\title{
Experimental Mg Ix photorecombination rate coefficient
}

\author{
S. Schippers ${ }^{1}$, M. Schnell ${ }^{2}$, C. Brandau ${ }^{1}$, S. Kieslich ${ }^{1}$, A. Müller ${ }^{1}$, and A. Wolf ${ }^{2}$ \\ 1 Institut für Atom- und Molekülphysik, Justus-Liebig-Universität Giessen, Leihgesterner Weg 217, 35392 Giessen, Germany \\ 2 Max-Planck-Institut für Kernphysik, Saupfercheckweg 1, 69117 Heidelberg, Germany
}

Received 3 March 2004 / Accepted 2 April 2004

\begin{abstract}
The rate coefficient for radiative and dielectronic recombination of beryllium-like magnesium ions was measured with high resolution at the Heidelberg heavy-ion storage ring TSR. In the electron-ion collision energy range 0-207 eV resonances due to $2 \mathrm{~s} \rightarrow 2 \mathrm{p}(\Delta N=0)$ and $2 \mathrm{~s} \rightarrow 3 l(\Delta N=1)$ core excitations were detected. At low energies below $0.15 \mathrm{eV}$ the recombination rate coefficient is dominated by strong $1 s^{2}\left(2 s 2 p{ }^{3} \mathrm{P}\right) 7 l$ resonances with the strongest one occuring at an energy of only $21 \mathrm{meV}$. These resonances decisively influence the $\mathrm{Mg}$ IX recombination rate coefficient in a low temperature plasma. The experimentally derived $\mathrm{Mg}$ IX dielectronic recombination rate coefficient ( $\pm 15 \%$ systematical uncertainty) is compared with the recommendation by Mazzotta et al. (1998, A\&AS, 133, 403) and the recent calculations by Gu (2003, ApJ, 590, 1131) and by Colgan et al. (2003, A\&A, 412, 597). These results deviate from the experimental rate coefficient by $130 \%, 82 \%$ and $25 \%$, respectively, at the temperature where the fractional abundance of $\mathrm{Mg}$ IX is expected to peak in a photoionized plasma. At this temperature a theoretical uncertainty in the $1 s^{2}\left(2 s 2 p{ }^{3} \mathrm{P}\right) 7 l$ resonance positions of only $100 \mathrm{meV}$ would translate into an uncertainty of the plasma rate coefficient of almost a factor 3 . This finding emphasizes that an accurate theoretical calculation of the $\mathrm{Mg}$ IX recombination rate coefficient from first principles is challenging.
\end{abstract}

Key words. atomic data - atomic processes - line: formation - plasmas - radiation mechanisms: general

\section{Introduction}

For the accurate calculation of the ionization equilibrium in astrophysical plasmas, rate coefficients for the population and depopulation of the various ion charge states have to be known precisely. To date most of the required rate coefficients are derived from theoretical calculations, and, hence, experimental benchmarks are required for testing and improving the theoretical methods. This is especially true for recombination in photoionized plasmas, which occurs at relatively low plasma temperatures of only a few electron volts. At such low temperatures the recombination rate coefficient, depending on the ion under consideration, can strongly be influenced by the existence of low-energy dielectronic recombination (DR) resonances that are difficult to theoretically predict with sufficient accuracy.

Experimentally derived plasma rate coefficients were previously published for the recombination of C IV (Schippers et al. 2001), O VI (Böhm et al. 2003), Ti V (Schippers et al. 1998), Ni XVIII (Fogle et al. 2003), Ni XXVI (Schippers et al. 2000) and Fe XVIII-Fe XXII (Savin et al. 1997, 1999, 2002a,b, 2003). Here the Mg IX recombination rate coefficient derived from experimental measurements at a heavy ion storage ring is provided.

Send offprint requests to: $\mathrm{S}$. Schippers, e-mail: Stefan.E.Schippers@strz.uni-giessen.de
The approximate temperature ranges where beryllium-like magnesium forms in photoionized and in collisionally ionized plasmas can be obtained from the work of Kallman \& Bautista (2001) who calculated the fractional abundances of ions in plasmas for a variety of physical conditions. For photoionized plasmas they find that the fractional $\mathrm{Mg}$ IX abundance peaks at an ionization parameter of $\log \zeta=0.9$ corresponding to a temperature of about $2.8 \mathrm{eV}$. The "photoionized zone" may be defined as the range of temperatures where the fractional abundance of a given ion exceeds $10 \%$ of its peak value. For Mg IX this corresponds to the temperature range $2-13 \mathrm{eV}$. Using the same criterion and the results of Kallman \& Bautista (2001) for coronal equilibrium the Mg IX "collisionally ionized zone" is estimated to extend over the temperature range $60-170 \mathrm{eV}$. It should be kept in mind that these temperature ranges are only indicative. In particular, they depend on the accuracy of the atomic data base used by Kallman \& Bautista (2001) and, in case of the photoionization zone, on the assumed $1 / E$ energy dependence of the ionizing radiation. Nevertheless, the above given temperature ranges will be used in the discussion below.

From the present measurements it is found that the Mg IX recombination rate coefficient in the photoionization zone is decisively influenced by the presence of a strong DR resonance at an electron-ion collision energy of $21 \mathrm{meV}$. Any theoretical calculation aiming at an accurate $\mathrm{Mg}$ IX rate coefficient will have to predict this resonance's position with 
an error of less than a few meV. Presently available atomicstructure computer codes are generally not capable of providing results with such an accuracy ab initio. In the standard theoretical approaches the situation is often improved by using spectroscopically observed target energies for the atomic structure (Colgan et al. 2003; Gu 2003a). As will be shown below, a $100 \mathrm{meV}$ uncertainty on the theoretical resonance position would translate into an uncertainty of the Mg IX DR rate coefficient of a factor 2.7 at the temperature where the Mg IX abundance is expected to peak in photoionization equilibrium.

\section{Experiment}

Various aspects of recombination measurements at the heavy-ion storage ring TSR of the Max-Planck-Institut für Kernphysik (MPI-K) in Heidelberg, Germany, have been described by Kilgus et al. (1992), Lampert et al. (1996), Pastuszka et al. (1996), Müller \& Wolf (1997), and Schippers et al. (2000, 2001). For the present experiment a MgIX ion beam with an energy of about $4.2 \mathrm{MeV} / \mathrm{u}$ was provided by the MPI-K linear accelerator facility and injected into the storage ring. There, over a distance of $\sim 1.5 \mathrm{~m}$, the ion beam was merged with a collinearly moving, magnetically guided electron beam. The electron beam served two purposes. First, it acted as a coolant for the ion beam, i.e., the ion beam's diameter and momentum spread were reduced by collisions with the much "colder" electrons (electron cooling, Poth 1990). Second, the electron cooling device (electron cooler) was subsequently used as an electron target where the ions recombined with the electrons and thereby changed their charge state. After adjusting the electron energy to a well defined value by tuning the electron cooler's cathode voltage appropriately, recombined ions were separated from the circulating parent-ion beam in the first bending magnet downstream from the electron cooler. Because of their high velocity they were efficiently counted with a scintillation detector. Absolute recombination rate coefficients as a function of relative energy between electrons and ions were derived from the measured count rate by i) subtracting the separately measured "background" count rate due to electron capture from residual gas molecules and by ii) normalizing to the number of stored ions and to the electron current which were both continuously monitored during the measurement. The "merged-beams technique" was recently reviewed by Phaneuf et al. (1999).

For the derivation of absolute rate coefficients from merged electron-ion beams measurements the contamination of the ion beam by metastable ions is an issue of concern. In a storage-ring experiment metastable ions can usually be given enough time (up to a few seconds) to decay before data taking is started. However, berylliumlike ions with zero nuclear spin like ${ }^{24} \mathrm{Mg}$ IX possess an extremely long-lived metastable $1 \mathrm{~s}^{2} 2 \mathrm{~s} 2 \mathrm{p}^{3} \mathrm{P}_{0}$ state which cannot decay to the $1 \mathrm{~s}^{2} 2 \mathrm{~s}^{2}{ }^{1} \mathrm{~S}_{0}$ ground state by a one-photon transition. DR resonances due to the excitation of the ${ }^{3} \mathrm{P}_{0}$ metastable state were observed in single-pass merged-beams experiments with berylliumlike ions by Badnell et al. (1991). Such resonances are not observed in the present experiment. All measured resonances can be attributed to the excitation of Mg IX ground-state ions.

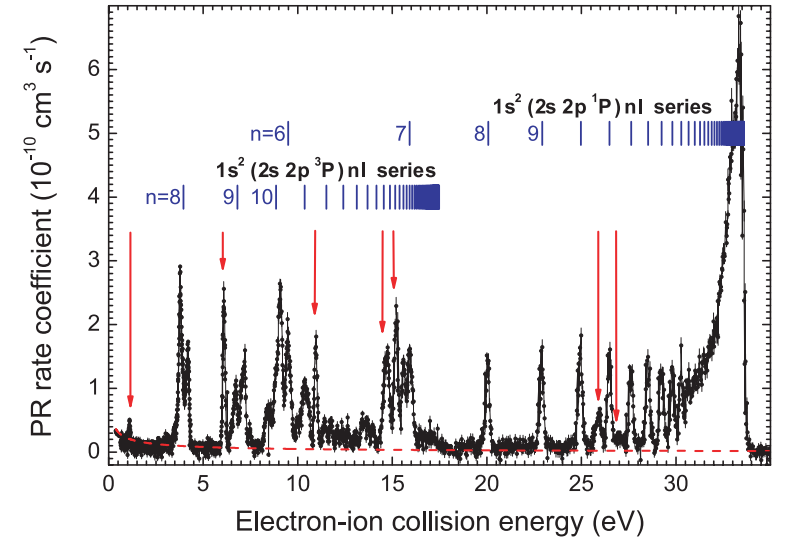

Fig. 1. Measured Mg IX merged-beams recombination rate-coefficient in the electron-ion collision energy region of DR $1 \mathrm{~s}^{2} 2 \mathrm{~s} 2 \mathrm{p} n l$ resonances attached to $1 \mathrm{~s}^{2} 2 \mathrm{~s}^{2} \rightarrow 1 \mathrm{~s}^{2} 2 \mathrm{~s} 2 \mathrm{p}(\Delta N=0)$ core excitations. The dashed line is the calculated hydrogenic rate coefficient for radiative recombination (RR). Positions of some $1 \mathrm{~s}^{2} 2 \mathrm{p}^{2} n l$ TR resonances are marked by vertical arrows (see text).

\section{Results}

The experimental Mg IX merged-beams recombination rate coefficient is shown in Figs. 1-3 over different ranges of electronion collision energy. Figure 1 displays all measured recombination resonances due to $1 \mathrm{~s}^{2} 2 \mathrm{~s}^{2} \rightarrow 1 \mathrm{~s}^{2} 2 \mathrm{~s} 2 \mathrm{p}$ and $1 \mathrm{~s}^{2} 2 \mathrm{~s}^{2} \rightarrow$ $1 \mathrm{~s}^{2} 2 \mathrm{p}^{2}(\Delta N=0)$ core excitations with the exception of the resonances at very low energies below $0.15 \mathrm{eV}$. These were measured separately with higher resolution and are displayed in Fig. 2. Finally, Fig. 3 shows at higher energies the DR spectrum for resonances attached to $1 \mathrm{~s}^{2} 2 \mathrm{~s}^{2} \rightarrow 1 \mathrm{~s}^{2} 2 \mathrm{~s} 3 l(\Delta N=1)$ core excitations.

\section{1. $\Delta N=0$ di- and trielectronic recombination}

Most of the measured $\Delta N=0$ photorecombination resonances in Fig. 1 can be ascribed to $1 \mathrm{~s}^{2} 2 \mathrm{~s}^{2} \rightarrow 1 \mathrm{~s}^{2}\left(2 \mathrm{~s} 2 \mathrm{p}{ }^{3} \mathrm{P}\right)$ and $1 s^{2} 2 s^{2} \rightarrow 1 s^{2}\left(2 s 2 p^{1} P\right)$ excitations. The corresponding Rydberg series of DR resonances converge to their respective series limits. Their spectroscopic values are 17.56 and $33.685 \mathrm{eV}$ (Martin et al. 1999). In order to match the experimental series limits - obtained by extrapolating the $1 \mathrm{~s}^{2} 2 \mathrm{~s} 2 \mathrm{p} n l$ resonance positions to $n \rightarrow \infty$ - to these values a scaling factor 1.00564 was applied to the energy scale. This factor is within the experimental uncertainty.

Next to the $1 \mathrm{~s}^{2} 2 \mathrm{~s} 2 \mathrm{p} n l$ DR resonances, further resonances are attached to $2 s^{2} \rightarrow 2 p^{2}$ double core excitations; they appear especially below the $1 \mathrm{~s}^{2}\left(2 \mathrm{~s} 2 \mathrm{p}{ }^{3} \mathrm{P}\right)$ series limit. The radiative decay of these triply excited resonance states to below the Mg IX ionization limit completes trielectronic recombination (TR). The importance of TR for the photorecombination of berylliumlike ions was only recently discovered and discussed by Schnell et al. (2003). For berylliumlike Cl XIV they find that over the plasma temperature range $1-100 \mathrm{eV}$ TR contributes up to $20-40 \%$ to the total recombination rate coefficient.

The disentanglement of TR and DR contributions to the measured recombination spectrum requires detailed atomic 


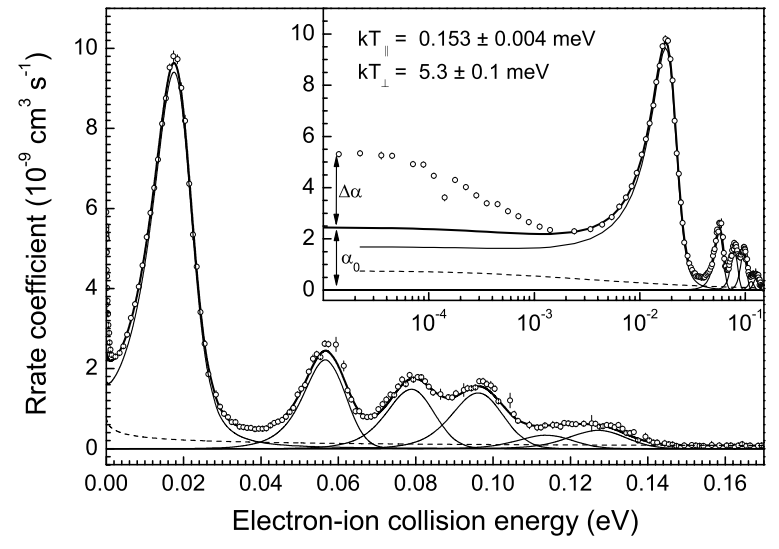

Fig. 2. High resolution Mg IX merged-beams recombination ratecoefficient in the energy range of of the $1 s^{2}\left(2 s 2 p{ }^{3} \mathrm{P}\right) 7 l$ DR resonances close to zero electron-ion collision energy: experiment (symbols) and RR and DR resonance fit (thick full line). Individual contributions to the fit are 6 DR resonances (thin full lines) and RR (thin dashed line). Data points below $3 \mathrm{meV}$ were excluded from the fit. The electron beam temperatures resulting from the fit are indicated in the inset which shows the same data on a logarithmic energy scale. It emphasizes the excess recombination rate coefficient $\Delta \alpha$ at very low energies (see text). The maximum enhancement is $\left(\Delta \alpha+\alpha_{0}\right) / \alpha_{0} \approx 2.3$. Further fit results are given in Table 1.

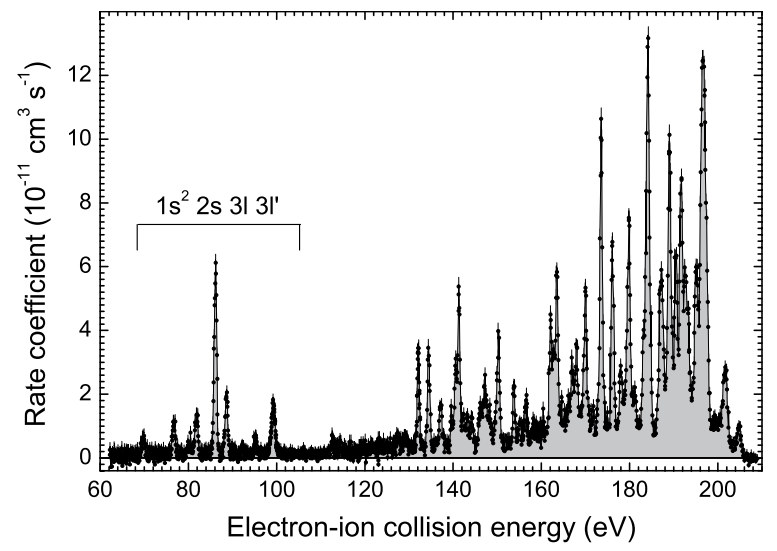

Fig. 3. Measured Mg IX merged-beams recombination rate-coefficient in the electron-ion collision energy region of DR $1 \mathrm{~s}^{2} 2 \mathrm{~s} 3 \mathrm{lnl} l^{\prime}$ resonances attached to $1 \mathrm{~s}^{2} 2 \mathrm{~s}^{2} \rightarrow 1 \mathrm{~s}^{2} 2 \mathrm{~s} 3 l(\Delta N=1)$ core excitations.

structure calculations and is beyond the scope of this paper. Therefore, only obvious TR contributions are marked in Fig. 1. For a more detailed discussion of TR the reader is referred to the work of Schnell et al. (2003). For the present purpose of deriving the experimental Mg IX plasma rate coefficient the question of the origin of individual resonances is not relevant. In any theoretical calculation, however, TR has to be accounted for in order to arrive at an accurate Mg IX plasma rate coefficient.

\subsection{Recombination at low energies}

Figure 2 shows the experimental merged-beams Mg IX recombination rate coefficient from very low energies up to $0.17 \mathrm{eV}$. In this energy range, the recombination rate coefficient is dominated by $1 \mathrm{~s}^{2}\left(2 \mathrm{~s} 2 \mathrm{p}{ }^{3} \mathrm{P}\right) 7 \mathrm{l}$ DR resonances. For an accurate determination of the resonance parameters we fitted 6 DR resonance line profiles to the measured spectrum as well as a smooth contribution due to radiative recombination (RR). In the fit the corresponding cross sections $\sigma(E)$ were convoluted with the experimental electron energy distribution

$$
\begin{aligned}
f\left(E, \hat{E}, T_{\|}, T_{\perp}\right) & =\frac{1}{k_{\mathrm{B}} T_{\perp} \xi} \exp \left(-\frac{E-\hat{E} / \xi^{2}}{k_{\mathrm{B}} T_{\perp}}\right) \\
\times & {\left[\operatorname{erf}\left(\frac{\sqrt{E}+\sqrt{\hat{E}} / \xi^{2}}{\sqrt{k_{\mathrm{B}} T_{\|}} / \xi}\right)+\operatorname{erf}\left(\frac{\sqrt{E}-\sqrt{\hat{E}} / \xi^{2}}{\sqrt{k_{\mathrm{B}} T_{\|}} / \xi}\right)\right] . }
\end{aligned}
$$

It is characterized by the longitudinal and transversal - with respect to the electron beam direction - temperatures $T_{\|}$and $T_{\perp}$, respectively (Kilgus et al. 1992), and $\xi=\left(1-T_{\|} / T_{\perp}\right)^{1 / 2}$. Furthermore, $k_{\mathrm{B}}$ denotes Boltzmann's constant and $\operatorname{erf}(x)=$ $2 \pi^{-1 / 2} \int_{0}^{x} \exp -t^{2} \mathrm{~d} t$ is the error function.

The convolution of the recombination cross section with the above experimental electron energy distribution yields the experimental merged-beams rate coefficient

$\alpha_{M B}(\hat{E})=\int_{0}^{\infty} \sqrt{2 E / m_{\mathrm{e}}} \sigma(E) f\left(E, \hat{E}, T_{\|}, T_{\perp}\right) \mathrm{d} E$

where $\hat{E}$ and $m_{\mathrm{e}}$ denote the experimental electron-ion collision energy and the electron rest mass, respectively. For each DR resonance the cross section was taken to be

$\sigma^{(\mathrm{DR})}(E)=\frac{\bar{\sigma}}{\pi} \frac{E_{\mathrm{res}}}{E} \frac{\Gamma / 2}{\left(E-E_{\mathrm{res}}\right)^{2}+(\Gamma / 2)^{2}}$.

The DR line shape is assumed to be a Lorentzian multiplied by a factor $E_{\mathrm{res}} / E$ accounting for the $1 / E$ dependence of the DR cross section at low energies (Schippers et al. 1998). For $E_{\text {res }} \gg \Gamma$ this factor can be neglected. In the limit $\Gamma \rightarrow 0$ the DR-resonance profile becomes a delta-function and the corresponding merged-beams rate coefficient can be expressed analytically as

$\alpha_{\mathrm{MB}}^{(D R)}(\hat{E})=\bar{\sigma} \sqrt{2 E_{\mathrm{res}} / m_{\mathrm{e}}} f\left(E_{\mathrm{res}}, \hat{E}, T_{\|}, T_{\perp}\right)$

with $f\left(E_{\mathrm{res}}, \hat{E}, T_{\|}, T_{\perp}\right)$ from Eq. (1). For $\Gamma>0$ the mergedbeams rate coefficient $\alpha_{\mathrm{MB}}^{(D R)}(\hat{E})$ was evaluated numerically.

For the radiative recombination (RR) cross section the semi-classical hydrogenic formula of Bethe \& Salpeter (1957) was used in slightly modified form, i.e.,

$$
\sigma^{(R R)}(E)=2.105 \times 10^{-22} \mathrm{~cm}^{2} \sum_{n_{\min }}^{n_{\max }} k_{n} t_{n} \frac{\left(Z_{\mathrm{eff}}^{2} \mathcal{R}\right)^{2}}{n E\left(Z_{\mathrm{eff}}^{2} \mathcal{R}+n^{2} E\right)}
$$

and the convolution (Eq. (2)) was performed numerically. In Eq. (5) the letter $\mathcal{R}$ denotes the Rydberg constant. The effective charge was taken to be $Z_{\text {eff }}=8$ and the summation over the principal quantum numbers $n$ was carried out from $n_{\min }=2$ to $n_{\max }=44$. The latter value is determined by field ionization in the charge-state-analyzing dipole magnet (Schippers et al. 2001). The constants $t_{n}$ account for partly filled shells. Here $t_{2}=6 / 8$ and $t_{n}=1$ for $n>2$ were used. The factors $k_{n}$ correct for the deviation of the semi-classical cross sections from 
the more exact quantum mechanical results and were calculated following the prescription of Andersen \& Bolko (1990). They are monotonically increasing for increasing $n$, starting from $k_{2}=0.877$ and approaching unity for higher $n$.

The resonance parameters that were obtained from the fit are listed in Table 1. The reduced $\chi^{2}$ of the fit is 1.24 . The electron beam temperatures resulting from the fit are $k_{\mathrm{B}} T_{\|}=$ $0.153 \pm 0.004 \mathrm{meV}$ and $k_{\mathrm{B}} T_{\perp}=5.1 \pm 0.1 \mathrm{meV}$. These values roughly correspond to what is expected from the electron cooler settings (Pastuszka et al. 1996). Data points below $3 \mathrm{meV}$ were excluded from the fit. At lower energies the measured rate coefficient exceeds the fitted one by a factor of up to 2.3 (inset of Fig. 2). This recombination rate enhancement at very low energies is an inherent feature of merged-beams experiments at electron coolers (Gwinner et al. 2000; Heerlein et al. 2002; Hörndl et al. 2003; Wolf \& Gwinner 2003). In the present context, this recombination rate enhancement can be clearly distinguished from the normal photorecombination rate coefficient and is excluded from the experimentally derived plasma rate coefficient of Sect. 3.4.

\section{3. $\Delta N=1$ dielectronic recombination}

Resonances attached to $1 \mathrm{~s}^{2} 2 \mathrm{~s}^{2} \rightarrow 1 \mathrm{~s}^{2} 2 \mathrm{~s} 3 l(\Delta N=1)$ core excitations occur in the energy range $70-205 \mathrm{eV}$ (Fig. 3). The lowest resonances of this group are the $1 \mathrm{~s}^{2} 2 \mathrm{~s} 3 l 3 l^{\prime}$ DR resonances extending to energies of up to about $100 \mathrm{eV}$. The higher$n$ manifolds of $1 \mathrm{~s}^{2} 2 \mathrm{~s} 3 l n l^{\prime}$ DR resonances mutually overlap. In contrast to $\Delta N=0 \mathrm{DR}$, the resonance strengths of the $\Delta N=$ 1 DR resonances rapidly decrease with increasing $n$. Therefore, the resonance strength does not pile up at the series limits. In Fig. 3 two series limits can be discerned at 202.25 and $205.14 \mathrm{eV}$. In order to match these values from the NIST atomic spectra data base (Martin et al. 1999) for the $1 \mathrm{~s}^{2} 2 \mathrm{~s} 3 \mathrm{~d}^{1} \mathrm{D} n l$ and the $1 \mathrm{~s}^{2} 2 \mathrm{~s} 3 \mathrm{~d}^{3} \mathrm{D} n l$ series, respectively, the experimental energy scale was multiplied by a factor 1.0091 . This rescaling is within the experimental uncertainty.

\subsection{Plasma recombination rate coefficient}

Formally, the recombination rate-coefficient in a plasma is obtained by using an isotropic Maxwellian distribution function in Eq. (2), i.e.,

$$
\alpha_{\text {plasma }}\left(k_{\mathrm{B}} T\right)=\frac{4}{\sqrt{2 \pi m_{\mathrm{e}}}\left(k_{\mathrm{B}} T\right)^{3 / 2}} \int_{0}^{\infty} \sigma(E) E \exp \left(-\frac{E}{k_{\mathrm{B}} T}\right) \mathrm{d} E
$$

For plasma temperatures $k_{\mathrm{B}} T \gg k_{\mathrm{B}} T_{\perp}$ a factor $\sigma(E) \sqrt{2 E / m_{\mathrm{e}}}$ in the integral may be replaced by the experimental mergedbeams recombination rate coefficient. This approach can safely be used for all recombination resonances with $E_{\mathrm{res}} \gg k_{\mathrm{B}} T_{\perp}$. Here it was applied to all recombination resonances above $0.2 \mathrm{eV}$ that are shown in Figs. 1 and 3. In order to exclude any effect of the finite experimental energy spread on the experimentally derived plasma rate coefficient, Eq. (6) was used with the resonance cross section from Eq. (3) for the resonances at lower energies. The contribution of the recombination resonances below $0.2 \mathrm{eV}$ (Fig. 2) to the plasma recombination
Table 1. Results of fitting individual DR resonances to the measured recombination rate coefficient at low energies (Fig. 2). Each resonance is characterized by its resonance position $E_{\text {res }}$, width $\Gamma$ and strength $\bar{\sigma}$. Numbers in brackets denote the statistical uncertainties obtained from the fit. If no value for the width is given a delta-function-like resonance was assumed.

\begin{tabular}{clc}
\hline \hline$E_{\mathrm{res}} /[\mathrm{meV}]$ & $\Gamma /[\mathrm{meV}]$ & $\bar{\sigma} /\left[10^{-18} \mathrm{eV} \mathrm{cm}^{2}\right]$ \\
\hline $20.92(5)$ & $4.6(1)$ & $17.93(7)$ \\
$60.2(1)$ & - & $2.23(2)$ \\
$82.5(1)$ & - & $1.41(2)$ \\
$99.9(2)$ & - & $1.29(2)$ \\
$117.6(9)$ & - & $0.30(2)$ \\
$131.0(4)$ & - & $0.41(2)$ \\
\hline
\end{tabular}

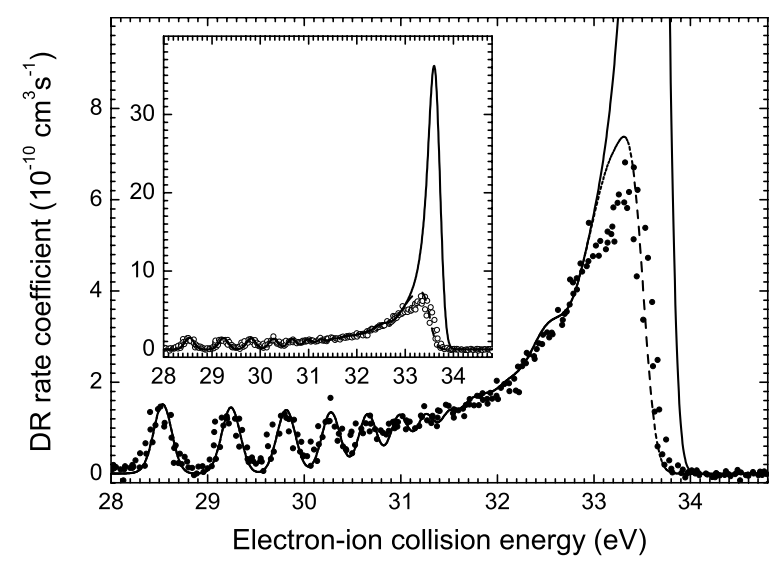

Fig. 4. Experimental merged-beams recombination rate coefficient in the region of high- $n 1 \mathrm{~s}^{2}\left(2 \mathrm{~s} 2 \mathrm{p}{ }^{1} \mathrm{P}\right) n l \mathrm{DR}$ resonances together with the result of an AUTOSTRUCTURE calculation scaled by a factor 0.7 . The dashed line is the theoretical result with account for the experimental field ionization of high- $n$ Rydberg states using the fieldionization model of Schippers et al. (2001). The full curve is the theoretical result including the full DR resonance strength up to $n=1000$. The inset shows the same curves on a different scale for an overview.

rate-coefficient was evaluated by using the fitted resonance parameters from Table 1.

For the derivation of the experimental MgIX recombination rate coefficient one has to be aware of the fact that loosely bound high- $n$ Rydberg states are easily field ionized in our experimental setup. This effect was modelled in considerable detail by Schippers et al. (2001). Generally, it is more significant for lower charged ions. As shown in Fig. 4 a theoretical calculation of the Mg IX DR rate coefficient using the AUTOSTRUCTURE code of Badnell (1986) can only be reconciled with the experimental data if field-ionization is taken into account.

In the present case the effect of field-ionization on the measured DR spectrum can be approximately described as a cutoff of high- $n$ Rydberg resonances with $n \gtrsim 44$. In order to account for the unmeasured DR resonance strength the DR spectrum for 
$n<44$ is extrapolated to higher $n$ by scaling the result of the AUTOSTRUCTURE calculation to the experimental spectrum in the energy range $28.0-32.9 \mathrm{eV}$ (scaling factor 0.7, Fig. 4) and by replacing the measured merged-beams rate coefficient by the scaled calculated value in the energy range 32.9-34.1 eV.

In principle, such a correction also has to be made for all other series of Rydberg resonances. For $\Delta N=0$ DR such other series are relatively weak (Fig. 1) and their cutoff by field-ionization does not lead to a significant error when considering the overall $\pm 15 \%$ experimental uncertainty of the absolute rate coefficient scale (Lampert et al. 1996). Regarding $\Delta N=1 \mathrm{DR}$, the resonance strength, as already mentioned, decreases strongly with increasing $n$, leading again to a negligible effect of the experimental field-ionization on the plasma rate coefficient.

Our experimentally derived Mg IX DR+TR recombination rate coefficient in a plasma - including the extrapolation of the $1 \mathrm{~s}^{2}\left(2 \mathrm{~s} 2 \mathrm{p}{ }^{1} \mathrm{P}\right) n l$ resonance strength to $n=1000$ (cf. Fig. 4) is shown in Fig. 5 as a thick full line. For the convenient use of our result in plasma modelling codes the following functional dependence - customarily used for DR rate coefficients - was fitted to our experimentally derived curve:

$\alpha_{\text {fit }}^{(\mathrm{DR})}=\frac{1}{T^{3 / 2}} \sum_{i} c_{i} \exp \left(-\frac{E_{i}}{k_{\mathrm{B}} T}\right)$.

During the fitting procedure the coefficients $c_{i}$ and $E_{i}$ were varied. The fit results are listed in Table 2.

In the fit to the low-energy experimental merged-beams recombination rate coefficient (Fig. 2) RR was also included (with $T_{\|}$and $T_{\perp}$ as the only free fit parameters and all other parameters kept fixed). The same RR cross section was used to draw the RR contribution in Fig. 1. This shows that Eq. (5) for the RR cross section is consistent with our experimental data. Therefore, Eq. (5), in conjunction with Eq. (6), can also be used to derive the experimental Mg IX RR rate coefficient in a plasma.

To this end, the RR cross section parameters were taken from Sect. 3.2. In order to make up for field-ionization effects the plasma RR rate coefficient in Fig. 5 (thin full line) was calculated with $n_{\max }=1000$ instead of $n_{\max }=44$ as in Fig. 2 . For convenient further use our Mg IX RR rate coefficient in a plasma was fitted by a formula introduced by Verner \& Ferland (1996):

$\alpha_{\text {fit }}^{(\mathrm{RR})}=A\left[\sqrt{\frac{T}{T_{0}}}\left(1+\sqrt{\frac{T}{T_{0}}}\right)^{1-b}\left(1+\sqrt{\frac{T}{T_{1}}}\right)^{1+b}\right]^{-1}$.

The parameter values that were obtained from the fit are $A=$ $5.7414 \times 10^{-10} \mathrm{~cm}^{3} \mathrm{~s}^{-1}, b=0.67692, T_{0}=206.15 \mathrm{~K}$, and $T_{1}=7.6200 \times 10^{6} \mathrm{~K}$. Over the temperature ranges $1-2.9 \times$ $10^{6} \mathrm{~K}$ and $2.9 \times 10^{6}-10^{8} \mathrm{~K}$ the fit is accurate to more than $2 \%$ and $3 \%$, respectively.

In Fig. 5 the present experimentally derived RR rate coefficient is compared with the the recent theoretical result of Gu (2003b, dotted line). The difference is less than $17 \%$ over the temperature range where $\mathrm{Mg}$ IX exists in a photoionized plasma.
Table 2. Parameters for the fit of Eq. (7) to the experimentally derived $\mathrm{Mg}$ IX DR+TR rate coefficient in a plasma. In the temperature ranges $25-350 \mathrm{~K}$ and $350-9 \times 10^{8} \mathrm{~K}$ the fit deviates less than $2 \%$ and $1 \%$, respectively, from the experimentally derived result. The systematic uncertainty of the experimentally derived absolute recombination rate coefficient is $\pm 15 \%$ (Lampert et al. 1996).

\begin{tabular}{ccc}
\hline \hline$i$ & $c_{i} /\left[\mathrm{cm}^{3} \mathrm{~s}^{-1} \mathrm{~K}^{3 / 2}\right]$ & $E_{i} /[\mathrm{eV}]$ \\
\hline 1 & $3.5960 \times 10^{-2}$ & $2.0071 \times 10^{6}$ \\
2 & $2.0109 \times 10^{-2}$ & $3.7011 \times 10^{5}$ \\
3 & $2.6244 \times 10^{-3}$ & $1.2038 \times 10^{5}$ \\
4 & $3.6203 \times 10^{-4}$ & $4.5163 \times 10^{4}$ \\
5 & $4.1121 \times 10^{-5}$ & $9.2881 \times 10^{2}$ \\
6 & $2.6915 \times 10^{-5}$ & $2.2683 \times 10^{2}$ \\
7 & $6.7347 \times 10^{-7}$ & $3.7786 \times 10^{1}$ \\
\hline
\end{tabular}

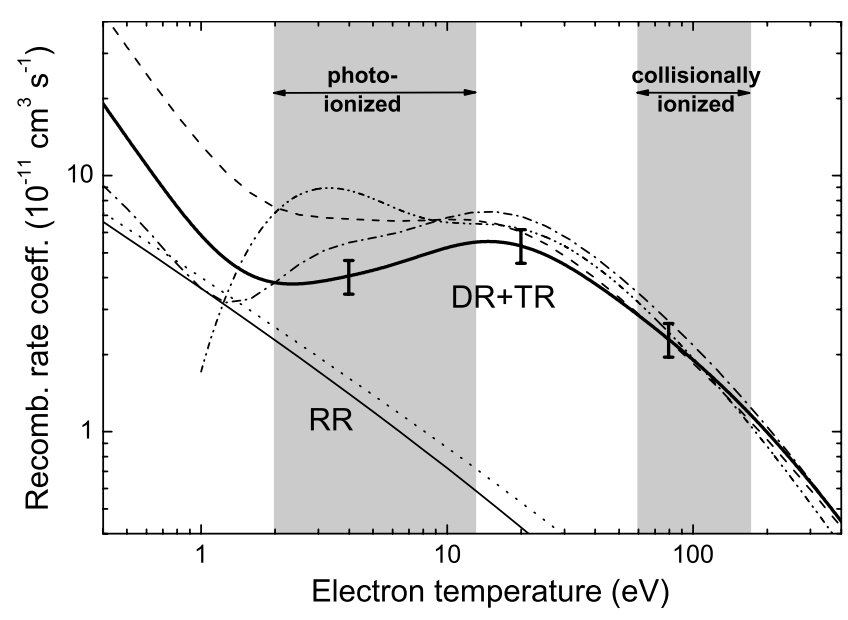

Fig. 5. Comparison of the present experimentally derived MgIX DR rate coefficient (thick full line) with the recommendation of Mazzotta et al. (1998, dash-dot-dotted line) and the theoretical results of Gu (2003a, dashed line) and Colgan et al. (2003, dash-dotted line). The error bars denote the $\pm 15 \%$ experimental uncertainty of the absolute rate coefficient. The thin full line is the RR rate coefficient calculated (Eq. (6)) with the RR cross section from Eq. (5) with $n_{\max }=1000$ and the remaining RR parameters from Sect. 3.2. The dotted line is the theoretical RR rate coefficient of $\mathrm{Gu}$ (2003b). The temperature ranges (see Sect. 1) where Mg IX exists in photoionized and in collisionally ionized plasmas, respectively, are highlighted.

The total (unified) Mg IX recombination rate coefficient is readily obtained as the sum of our DR+TR and RR rate coefficients. It should be noted that this result does not depend on the existence of possible quantum mechanical interferences between RR and DR, since interference has already been neglected during the decomposition of the measured spectrum into DR and RR (Fig. 2). In principle, interference between RR and DR can lead to asymmetric recombination resonance line shapes (see e.g., Schippers et al. 2002). No evidence for such effects is found in our experimental $\mathrm{MgIX}$ recombination 
spectra. The systematic uncertainty of the experimentally derived total $\mathrm{Mg} \mathrm{IX}$ recombination rate coefficient is $\pm 15 \%$ (Lampert et al. 1996).

\section{Discussion and conclusions}

In Fig. 5 the present experimentally derived DR+TR rate coefficient is compared with the recommendation of Mazzotta et al. (1998) and with the recent calculations of Gu (2003a) and Colgan et al. (2003). The recommended Mg IX DR rate coefficient of Mazzotta et al. (1998) deviates by up to $130 \%$ from the experimental result in the temperature range $2-13 \mathrm{eV}$ where Mg IX exists in photoionized plasmas. This rather large discrepancy questions the usefulness of the recommended Mg IX DR rate coefficient for the modeling of photoionized plasmas. In the temperature range $60-170 \mathrm{eV}$ (collisionally ionized zone) the recommended Mg IX DR rate coefficient of Mazzotta et al. (1998) agrees within 11\%, i.e., within the $15 \%$ experimental uncertainty, with the experimentally derived one.

In this temperature range the theoretical rate coefficient of Gu (2003a) shows very good agreement with the experimentally derived rate coefficient. The difference between the two curves in this range is less than $7 \%$. At lower temperatures, however, the discrepancy is up to $99 \%$ at $2 \mathrm{eV}$. At these low temperatures where Mg IX exists in photoionized plasmas, the theoretical result of Colgan et al. (2003) agrees better with the experimental rate coefficient. The deviation is less than $35 \%$ for $k_{\mathrm{B}} T>2 \mathrm{eV}$, less than $30 \%$ for $k_{\mathrm{B}} T>15 \mathrm{eV}$, and less than $15 \%$ for $k_{\mathrm{B}} T>100 \mathrm{eV}$. At the temperature $2.8 \mathrm{eV}$ where the fractional abundance of Mg IX is expected to peak in a photoionized plasma (Kallman \& Bautista 2001) the results of Gu (2003a) and Colgan et al. (2003) deviate from the experimental rate coefficient by $82 \%$ and $25 \%$, respectively.

The rather large low-temperature deviation of the theoretical rate coefficient of $\mathrm{Gu}$ (2003a) from the experimentally derived curve is most probably due to an inaccurate calculation of the DR resonance energies below $0.15 \mathrm{eV}$ (Fig. 2). At these very low energies the DR rate coefficient is very sensitive to slight variations of resonance positions. This is highlighted in Fig. 6 that displays the effect of hypothetical shifts of the low-energy resonance positions by $\pm 50 \mathrm{meV}$ and $\pm 100 \mathrm{meV}$ with respect to the tabulated values (Table 1) on the Mg IX DR rate coefficient. Obviously, a $\pm 100 \mathrm{meV}$ uncertainty of the low-energy $1 \mathrm{~s}^{2}\left(2 \mathrm{~s} 2 \mathrm{p}^{3} \mathrm{P}\right) 7 l$ resonance positions translates into an uncertainty of a factor 2.7 of the plasma rate coefficient in the temperature range where the fractional abundance of Mg IX peaks in a photoionized gas.

These findings demonstrate that an accurate calculation of DR rates for ions that exhibit DR resonances close to zero energy is challenging. In case of more complex ions this task is certainly beyond the capabilities of present-day atomic structure codes as is exemplified by a recent combined theoretical and experimental DR study of argon-like $\mathrm{Sc}^{3+}$ (Schippers et al. 2002). Recombination experiments at heavy-ion storage rings are certainly required for guiding the future development of the theoretical methods, especially in the case of low temperature DR rate coefficients for complex ions.

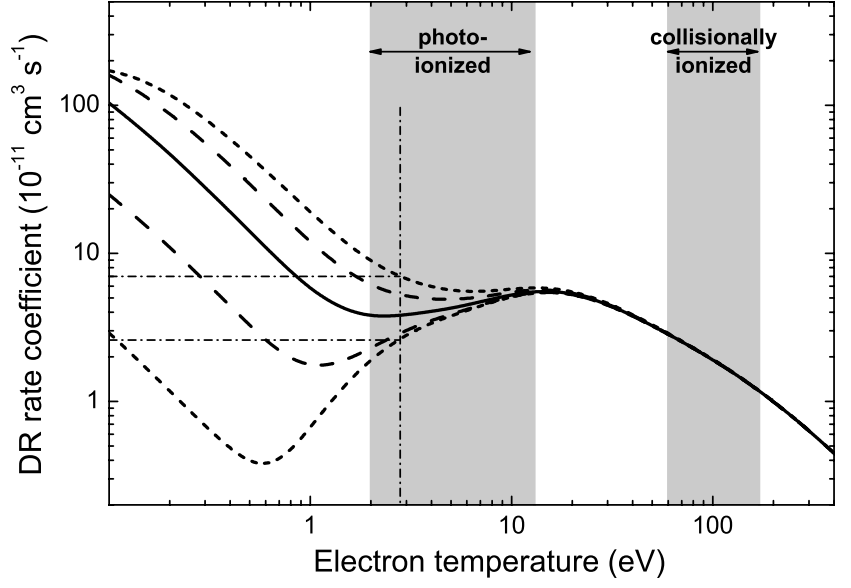

Fig. 6. Impact of a shift of the low energy $1 \mathrm{~s}^{2}\left(2 \mathrm{~s} 2 \mathrm{p}{ }^{3} \mathrm{P}\right) 7 \mathrm{l}$ resonances displayed in Fig. 2 on the Mg IX DR plasma rate-coefficient. The full curve is the experimental result, the long-dashed curves correspond to resonance shifts of $\Delta E_{\text {res }}= \pm 50 \mathrm{meV}$, and the short-dashed curves to a shift of $\Delta E_{\text {res }}= \pm 100 \mathrm{meV}$. Thereby, the lower curves correspond to resonance shifts to lower energies. The vertical dash-dotted line marks the temperature $2.8 \mathrm{eV}$ where the fractional abundance of Mg IX peaks in a photoionized gas (Kallman \& Bautista 2001). The horizontal dash-dotted lines indicate the factor 2.7 uncertainty of the plasma rate coefficient at this temperature that would be introduced by a $\pm 100 \mathrm{meV}$ uncertainty of the low energy resonance positions.

Acknowledgements. We thank D. W. Savin for helpful discussions and the MPI-K accelerator crew, in particular R. Repnow and M. Grieser, for their excellent support. This work was partly funded by Deutsche Forschungsgemeinschaft under contract Mu 1068/8.

\section{References}

Andersen, L. H., \& Bolko, J. 1990, Phys. Rev. A, 42, 1184

Badnell, N. R. 1986, J. Phys. B, 19, 3827,

http://amdpp.phys.strath.ac.uk/autos/

Badnell, N. R., Pindzola, M. S., Andersen, L. H., Bolko, J., \& Schmidt, H. T. 1991, J. Phys. B, 24, 4441

Bethe, H. A., \& Salpeter, E. E. 1957, Quantum Mechanics of Oneand Two-Electron Atoms (Berlin: Springer)

Böhm, S., Müller, A., Schippers, S., et al. 2003, A\&A, 405, 1157

Colgan, J., Pindzola, M. S., Whiteford, A. D., \& Badnell, N. R. 2003, A\&A, 412, 597

Fogle, M., Badnell, N. R., Eklöw, N., Mohamed, T., \& Schuch, R. 2003, A\&A, 409, 781

Gu, M. F. 2003a, ApJ, 590, 1131

Gu, M. F. 2003b, ApJ, 589, 1085

Gwinner, G., Hoffknecht, A., Bartsch, T., et al. 2000, Phys. Rev. Lett., 84,4822

Heerlein, C., Zwicknagel, G., \& Toepffer, C. 2002, Phys. Rev. Lett., 89,083202

Hörndl, M., Yoshida, S., Tökési, K., \& Burgdörfer, J. 2003, Hyperfine Interact., 146/147, 13

Kallman, T., \& Bautista, M. 2001, ApJS, 133, 221

Kilgus, G., Habs, D., Schwalm, D., et al. 1992, Phys. Rev. A, 46, 5730

Lampert, A., Wolf, A., Habs, D., et al. 1996, Phys. Rev. A, 53, 1413 
Martin, W. C., Sugar, J., Musgrove, A., et al. 1999, NIST Atomic Spectra Data Base, 2nd ed., National Institute of Standards and Technology, Gaithersburg, Maryland 20899-3460, USA, http://physics.nist.gov/cgi-bin/AtData/main_asd

Mazzotta, P., Mazzitelli, G., Colafrancesco, S., \& Vittorio, N. 1998, A\&A, 133, 403

Müller, A., \& Wolf, A. 1997, in Accelerator-based atomic physics techniques and applications, ed. J. C. Austin, \& S. M. Shafroth (Woodbury: AIP Press), 147

Pastuszka, S., Schramm, U., Grieser, M., et al. 1996, Nucl. Instrum. Methods A, 369, 11

Phaneuf, R. A., Havener, C. C., Dunn, G. H., \& Müller, A. 1999, Rep. Prog. Phys., 62, 1143

Poth, H. 1990, Phys. Rep., 196, 135

Savin, D. W., Bartsch, T., Chen, M. H., et al. 1997, ApJ, 489, L115
Savin, D. W., Kahn, S. M., Linkemann, J., et al. 1999, ApJS, 123, 687

Savin, D. W., Behar, E., Kahn, S. M., et al. 2002a, ApJS, 138, 337

Savin, D. W., Kahn, S. M., Linkemann, J., et al. 2002b, ApJ, 576, 1098

Savin, D. W., Kahn, S. M., Gwinner, G., et al. 2003, ApJS, 147, 421

Schippers, S., Bartsch, T., Brandau, C., et al. 1998, J. Phys. B, 31, 4873

Schippers, S., Bartsch, T., Brandau, C., et al. 2000, Phys. Rev. A, 62, 022708

Schippers, S., Kieslich, S., Müller, A., et al. 2002, Phys. Rev. A, 65, 042723

Schippers, S., Müller, A., Gwinner, G., et al. 2001, ApJ, 555, 1027

Schnell, M., Gwinner, G., Badnell, N. R., et al. 2003, Phys. Rev. Lett., 91, 043001

Verner, D. A., \& Ferland, G. J. 1996, ApJS, 103, 467

Wolf, A., \& Gwinner, G. 2003, Hyperfine Interact., 146/147, 5 